\title{
Écologie ou Umwelt? Une revue historiographique des engagements écologistes et environnementalistes en France et en Allemagne
}

\section{Anahita Grisoni et Rosa Sierra}

\section{OpenEdition}

\section{Journals}

Édition électronique

URL : http://journals.openedition.org/ifha/7403

DOI : $10.4000 /$ ifha. 7403

ISSN : 2198-8943

Éditeur

IFRA - Institut franco-allemand (sciences historiques et sociales)

\section{Édition imprimée}

Date de publication : 1 décembre 2013

ISSN : 2190-0078

Référence électronique

Anahita Grisoni et Rosa Sierra, «Écologie ou Umwelt ? Une revue historiographique des engagements écologistes et environnementalistes en France et en Allemagne ", Revue de l'IFHA [En ligne], 5 | 2013, mis en ligne le 17 février 2014, consulté le 02 mai 2019. URL : http://journals.openedition.org/ ifha/7403 ; DOl : 10.4000/ifha.7403

Ce document a été généré automatiquement le 2 mai 2019.

(CIFHA 


\title{
Écologie ou Umwelt? Une revue historiographique des engagements écologistes et environnementalistes en France et en Allemagne
}

\author{
Anahita Grisoni et Rosa Sierra
}

1 En matière d'engagement social et politique face aux enjeux environnementaux et à l'écologie, les sciences humaines et sociales se caractérisent d'une part par la coexistence d'une multiplicité de définitions, et d'autre part par une multitude d'axes de recherche. Ce constat s'applique tout autant à l'engagement des acteurs sociaux (acteurs institutionnels, politiques, associatifs ou non) qu'au travail des experts et des membres de la communauté scientifique. De la même façon, l'équivocité des termes ne semble pas exclusivement liée à une expansion très actuelle et par là même diffuse des enjeux environnementaux et de l'écologie dans l'espace public: une exploration des transformations de ces notions à travers l'espace et le temps en France et en Allemagne pendant les dernières décennies conduit à remettre en question leur équivalence dans des contextes nationaux différents.

2 Cette interrogation se prête particulièrement à une comparaison franco-allemande qui peut s'appuyer sur des bases géographiques et politiques: ces pays partagent une frontière commune et s'impliquent tous deux activement dans la construction de l'Union européenne. Cette comparaison est d'autant plus pertinente en sciences humaines lorsque l'on s'intéresse aux enjeux environnementaux et à l'écologie. Depuis maintenant deux décennies le cercle restreint des chercheurs français qui consacrent leurs études à l'environnement et à l'écologie se penchent avec prédilection sur le cas allemand en analysant la spécificité ou en l'abordant dans une perspective comparative ${ }^{1}$. Le savoir scientifique français sur l'environnement se construit ainsi à partir d'une réflexion sur l'Allemagne. Dans cette perspective, les sociologues Danny Trom, Loïc Blondiaux et 
Guillaume Courty considèrent que l'écologisme se définit «à partir du chassé-croisé franco-allemand $»^{2}$.

3 Cette interrogation s'inscrit avant tout dans un questionnement d'ordre linguistique et porte sur les différences de sens des concepts entre les deux pays. Ainsi, tout comme les termes de "Nachhaltigkeit » et de "développement durable » revêtent un sens qui n'est pas interchangeable, on constate un usage différencié des notions de "Umwelt " et d'« environnement ».Alors qu'il existe une distinction claire en français entre les notions d'environnement et d'écologie ${ }^{3}$, il semblerait que la notion allemande de "Umwelt » recouvre en partie ces deux notions. Il en va de même pour d'autres termes appartenant au champ lexical de l'environnement qu'il est particulièrement malaisé de traduire. Dans un article portant sur le phénomène de la décroissance Hélène Blanc et Michel Bodet soulignent les difficultés à emprunter un équivalent en langue allemande qui soit véritablement reconnu comme tel ${ }^{4}$. Ces divergences révèlent une construction sociale différente de la nature ${ }^{5}$ qui correspond à une histoire nationale spécifique. Face à la diversité sémantique des notions ainsi qu'à la pluralité de leurs évolutions, on peut s'interroger sur le bien-fondé de cette conception. En s'appuyant sur les travaux de Lionel Charles et Bernard Kalaora, il s'agit ici de considérer la " dimension environnementale » comme une entité composée de la "diversité des expériences" reposant sur une " hétérogénéité des espace-temps " ${ }^{6}$. Dans cette perspective, les dimensions écologiques et environnementales singulières caractéristiques d'un territoire sont au centre de l'approche et constituent un élément essentiel pour saisir une posture et des engagements de nature politique.

4 Au-delà de cette compréhension des particularismes, ces différences ne sont ni structurelles ni permanentes mais tributaires de transformations dans l'espace et le temps et soumises à des évolutions en termes de représentations et de construction symbolique. Il est donc indispensable que les travaux de recherche en sciences humaines et sociales portant sur le succès ou les difficultés des enjeux environnementaux et écologiques puissent imposer dans l'opinion publique des différents pays européens une vision prenant en compte le caractère dynamique et historique de ces phénomènes.

5 Loin de cette posture compréhensive et analytique, les représentations courantes (opposant de manière caricaturale une Allemagne romantique à une France grossièrement cartésienne et scientiste) reposent sur l'idée que les voisins d'Outre-Rhin seraient, pour des raisons culturelles, plus enclins à intégrer la nature et le bien-être aux fondements de la vie en société. De la même façon, pour Tobias Chilla, les œuvres d'art romantiques sont généralement analysées comme une «idéalisation de la nature ${ }^{7}$ ». Dans cette perspective, l'Allemagne ferait office de leader en matière de «bonnes pratiques environnementales ", portées par les acteurs sociaux, mais aussi en termes de politiques publiques, comme l'a récemment montré la décision de la chancelière Angela Merkel de suspendre l'activité des centrales nucléaires. Tobias Chilla met en garde contre les dangers essentialistes de ces présupposés, enfermant l'Allemagne dans une identité « environnementaliste »:

6 «Le thème d'une évolution spécifiquement allemande de la protection de la nature revient pour ainsi dire à reprendre ce que l'on a si souvent dit de la relation particulière qu'entretiennent les Allemands avec la nature et la protection de la nature : une nature transfigurée par le romantisme, instrumentalisée par le national-socialisme, la popularité défaitiste du "Waldsterben », etc. Bien des aspects de ce thème de l'environnement et de la nature sont souvent utilisés pour illustrer et exprimer l'identité nationale du 
typiquement allemand. Mais il y a aussi le risque que cette argumentation populaire serve à asseoir des clichés et des légendes en laissant de côté un autre modèle d'interprétation » 8 .

7 Dans cet article nous tenterons de remettre en cause l'idée selon laquelle l'engagement social et politique en matière d'environnement et d'écologie serait plus important ${ }^{9}$ en Allemagne qu'en France, en soumettant à l'analyse l'attrait pour le « modèle allemand ». Cette interrogation développée dans ce travail commun ${ }^{10}$ est présente chez de nombreux auteurs à travers la perspective d'une référence à un ensemble de représentations et de pratiques plus ou moins institutionnalisées et qui semblent être perçues comme un "modèle allemand ", et motive des questionnements plus larges. Considérer les notions de « Umwelt » et d'environnement, d'écologie et de «Ökologie » ainsi que leur intégration au sein du système social permet d'appréhender une vision différenciée des situations nationales et rend possible une mise à distance de la posture d'expert, qui aurait pu conduire à évaluer les institutions françaises à partir de leur « retard » dans l'adoption de certaines mesures environnementales. Davantage que les faits, ce qui intéresse ici le chercheur est la manière dont ils sont interprétés: une plus grande sensibilité à l'environnement du côté allemand pourrait être également perçue comme une absence de capacité critique face à la construction sociale classique de ces enjeux dans le débat public.

8 L'attrait pour le «modèle allemand » doit être soumis à l'analyse. Au-delà d'une approche comparative systématique visant à établir un bilan point par point, nous nous interrogeons ici sur les spécificités de ce modèle tel qu'il a été décrit par des auteurs francophones et germanophones ${ }^{11}$. Celles-ci pourraient expliquer que l'Allemagne puisse faire figure de référence en France. Dans quelle mesure la manière dont l'engagement politique en faveur de l'environnement et de l'écologie tel qu'il est véhiculé dans l'histoire des idées et porté par les acteurs sociaux et les scientifiques peut-il apparaître comme exemplaire aux yeux des Français? Loin des présupposés culturalistes, cette interrogation repose sur l'hypothèse que la notion même de "Umwelt " implique davantage la dimension de "lien social » et les problématiques sociales qui lui sont sousjacentes que le terme d'«environnement». Plutôt que de constituer un champ de revendications à part entière distinct des luttes sociales ${ }^{12}$, la notion de «Umwelt » contient ces deux domaines du social. Au-delà de ces considérations linguistiques, ces différences transparaissent à travers la manière dont ces notions sont portées par les différents acteurs sociaux. Que représente le "modèle allemand» en matière d'engagement politique pour l'environnement à l'échelle des sciences humaines et sociales, et comment les auteurs l'analysent-ils? Une mise en abîme historique, effectuée à partir des travaux des deux dernières décennies, nous a permis de dégager trois champs d'engagement qui constitueront les parties de cet article : (1) les « opinions et pratiques en matière d'environnement $»^{13}(1)$, créatrices d'un discours et d'une pensée environnementalistes (2) et enfin les associations écologistes ${ }^{14}$ et les partis « verts » ${ }^{15}$ (3). Nous aborderons ces trois champs d'engagement en nous interrogeant sur les différences linguistiques des notions françaises et allemandes en lien avec les enjeux environnementaux, notamment celles d'écologie et de «Umwelt ». 


\section{Opinions, pratiques, représentations en matière d'environnement en Allemagne : de la conscience environnementale ("Umweltbewusstsein ») aux " convictions écologistes "}

\subsection{Réflexions générales : les critères pour évaluer la conscience environnementale}

Dans l'étude de la conscience environnementale ou "Umweltbewusstsein », les auteurs distinguent communément un «Umweltverhalten » (comportement ou bonnes pratiques environnementales) de "Umwelteinstellungen» (attitudes environnementalistes). Le premier terme correspond aux actions humaines que l'on peut observer, alors que le second désigne les opinions et représentations présentes dans le discours des acteurs (par exemple, lors d'entretiens semi-directifs), mais qui ne se traduisent pas nécessairement par des actions concrètes.

L'étude des «attitudes environnementalistes» et des «bonnes pratiques environnementales » soulève des interrogations. Pour Peter Preisendörfer l'analyse des pratiques en lien avec les enjeux environnementaux ne peut se limiter à une étude des actions individuelles concrètes (par exemple, dans la sphère politique). Le chercheur doit avant tout s'attacher à comprendre "l'état et le développement de la conscience environnementale et des comportements environnementaux dans la population ", en particulier les opinions, représentations et pratiques qui ne correspondent pas aux manifestations d'un militantisme mais qui jouent néanmoins un rôle face aux enjeux environnementaux ${ }^{16}$. Selon Peter Preisendörfer il n'est pas de protection de l'environnement possible sans l'engagement de la population, d'où la nécessité de telles études. Là apparaît l'importance de la différence entre l'observation du " comportement " et l'étude des "attitudes environnementalistes" car si les impacts de cette première notion sur la protection de l'environnement sont évidents, il n'en est pas de même pour la seconde. L'analyse du discours des acteurs ne se substitue par conséquent pas à celle des comportements, mais la complète.

11 Les attitudes environnementales peuvent être étudiées dans une double perspective : par rapport à leurs impacts sur la transformation des valeurs sociales, d'une part, et par rapport à leurs interactions avec le comportement, d'autre part ${ }^{17}$. Or les implications pragmatiques des valeurs étant peu mesurables, Peter Preisendörfer se concentre sur le second champ d'étude. Contrairement à ce que l'on pourrait penser, il n'existe pas de corrélation directe entre le comportement et les attitudes environnementalistes ${ }^{18}$. Ce constat rejoint celui du politiste Jean-Paul Bozonnet, pour qui il existe un « hiatus entre attitudes environnementalistes et comportements ${ }^{19}$. Selon Peter Preisendörfer les attitudes environnementalistes ne représentent pas la seule motivation des comportements liés à la protection environnementale (parce qu'elles sont déterminées par un ensemble d'autres facteurs), mais elles agissent comme les "compléments stabilisateurs » de ces comportements ${ }^{20}$. Elles se développent en outre dans un certain «climat d'opinion publique» qui pèse sur les décisions des acteurs politiques et économiques et peuvent les inciter à prendre mesures visant à protéger l'environnement ${ }^{21}$. 
12 Outre les concepts de conscience environnementale et de comportement environnemental, la notion de " convictions écologistes » s'impose aussi dans l'étude des engagements liés aux enjeux environnementaux. Contrairement aux deux premières notions précitées, les convictions écologistes, comme le souligne Jean-Paul Bozonnet, se comprennent essentiellement à l'échelle globale de la société et de sa transformation, et dans une dimension politique, à travers des actions concrètes ${ }^{22}$. Dans cette perspective, la conscience environnementale définie comme «un souci plus ou moins grand de protection de la nature et de défense de l'environnement [...] $»^{23}$ est liée à un « souci de la qualité de la vie dans son milieu proche " ainsi qu'à une «conscience planétaire de ces questions ${ }^{24}$, souvent dépourvus d'un caractère véritablement politique et/ ou social. Cette dimension politique relève davantage de la «transformation écologiste de la société » qui renvoie à un « changement du système économique, des relations à l'État, de la vie quotidienne ou des idées $»^{25}$. Elle concerne tant les «bonnes habitudes » de la vie quotidienne (économiser l'électricité, renoncer à sa voiture) que « la démarche militante de participation aux organisations et aux actions protestataires ${ }^{26}$. Les convictions écologistes envisagent avant tout l'écologisme comme une idéologie, ou une vision du monde, hissée au même niveau que d'autres convictions idéologiques telles que le libéralisme, le socialisme, l'extrême-droite, etc., ou religieuses. À cet égard, les notions d'écologie, d'écologisme et de convictions écologistes en langue française sont davantage porteuses d'une dimension politique que celle d'environnement, alors que ce serait plutôt le cas des termes de «Umweltverhalten» et de «Umweltbewusstsein »en allemand, paradoxalement traduits en français par « environnement ».

13 Or la différence entre la «conscience environnementale» et les "convictions écologistes » telle qu'elle est définie par Jean-Paul Bozonnet ne devrait pas être comprise comme une opposition des termes. Elle permettrait plutôt d'envisager un large spectre regroupant différents types d'acteurs dans la société : ceux qui s'approprient certaines attitudes ou certaines opinions environnementales, situées à un extrême, et ceux qui adoptent des convictions écologistes, à l'autre extrême, et dont les comportements sont plus proches d'acteurs politiques qui s'engagent dans le monde associatif. Cette lecture des différentes postures est notamment analysée par Céline Caro qui prend en considération les évolutions à plus long terme, en particulier le développement historique de la conscience environnementale qui conduit à l'émergence, au XX $\mathrm{XX}^{\mathrm{e}}$ siècle, de l'écologie politique et des partis écologistes. Cette perspective est néanmoins secondaire dans la mesure où elle cache la coexistence de formes différenciées de conscience, d'attitudes, d'opinions et de comportements, d'engagements et d'incohérences en matière d'écologie, de protection de la nature et de l'environnement parmi les acteurs sociaux et institutionnels à toutes les échelles dans l'époque actuelle.

\section{2. État des lieux du sujet en Allemagne : des racines historiques ?}

14 Menée en Allemagne entre 1991 et 1998 et portant explicitement sur la conscience environnementale et les comportements environnementaux, la recherche de Peter Preisendörfer "Umwelteinstellung und Umweltverhalten in Deutschland ${ }^{27}$ a abouti à l'identification d'un "problème environnemental » dont l'importance aurait diminué entre 1991 et 1998. L'auteur invoque plusieurs raisons pouvant expliquer cette interprétation. L'urgence de maintenir l'État social ou d'intensifier les mesures anticriminalité prime sur celle de la protection de l'environnement (a). La perspective de 
payer davantage pour la protection de l'environnement fait l'objet de réticences $(b)^{28}$. Les causes de cette baisse seraient donc liées à l'intensification de certains problèmes économiques, mais aussi à la perception d'une amélioration des conditions environnementales au cours des années $1990^{29}$. Pour l'auteur, cette amélioration concerne les problèmes qui se posent dans le milieu proche, à travers l'institution de lois, par exemple $^{30}$. En dépit d'autres tendances venant relativiser ces données ${ }^{31}$, nous pouvons en conclure que les préoccupations environnementales sont devenues moins prioritaires au cours des années 1990du fait de la montée d'autres problématiques sociales ${ }^{32}$.

Bien que la perception des problèmes réels semble avoir diminué, l'enquête de Preisendörfer met en évidence le fait que «la conscience environnementale de la population allemande reste à un haut niveau " ${ }^{33}$. La baisse de la " sensibilité écologique " s'expliquerait plutôt par le fait que les discussions publiques autour des enjeux environnementaux ont un caractère de moins en moins sensible ${ }^{34}$.L'auteur interprète cette évolution comme un signe positif dans la longue durée car elle pourrait permettre d'apporter des solutions à long terme ${ }^{35}$.Dans le même temps, le constat d'une baisse de la conscience environnementale n'est pas lié à un recul dans le champ des comportements. Si l'usage des transports publics reste encore problématique, les comportements en matière de recyclage ainsi que les pratiques de consommation et d'économie d'énergie se seraient améliorés ${ }^{36}$.

Dans deux études menées en 2002 et 2004 pour l'Agence fédérale de l'environnement ( Umweltbundesamt $)^{37}$, plusieurs grandes tendances ont été mises en lumière : alors que les médias s'interrogent en 2002 sur la baisse éventuelle de la conscience environnementale en Allemagne, la protection de l'environnement reste un sujet important pour la population, même si cela n'est plus prioritaire dans l'actualité politique ${ }^{38}$. Entre 1993 et $2002^{39}$ l'importance des enjeux environnementaux dans la population a été sujette à des variations : en 1993 ils constituaient le premier sujet d'inquiétude des Allemands avant de connaître un recul en 1998/1999 et de remonter en 2002 au second rang des préoccupations. Tout au long de cette période, le chômage et l'économie représentent les enjeux les plus importants dans l'actualité politique et l'opinion publique ${ }^{40}$. Pourtant, si l'on considère qu'aujourd'hui l'écologie et l'économie ne sont pas complètement dissociables, cette analyse diachronique prend un tout autre sens puisque l'on ne peut plus simplement affirmer une priorité des enjeux économiques sur les enjeux environnementaux ${ }^{41}$. L'étude de 2004 souligne que, cette année-là, la sensibilité face aux enjeux environnementaux aurait augmenté par rapport à 2002.

Si la conscience environnementale des Allemands reste forte, il n'en demeure pas moins que les sujets concrets qui entrent dans la définition générale de cette notion changent: ainsi, dans les années 2000, les préoccupations des Allemands concernent moins les catastrophes environnementales que le changement climatique et le développement durable (cette notion étant comprise comme une question d'équité, de justice globale et intergénérationnelle ${ }^{42}$. Cette évolution des thématiques permet d'expliquer en partie l'effacement relatif des enjeux environnementaux dans la sphère de l'actualité politique : la perception de ces enjeux en termes de "catastrophes " ou de problèmes ayant des conséquences directes dans la vie des individus justifiait une urgence à agir et à gérer ces événements pour le politique ${ }^{43}$. La population aurait perçu cette gestion politique des menaces environnementales comme une capacité à apporter des solutions, engendrant ainsi progressivement un relâchement des préoccupations en matière d'environnement. Les auteurs qualifient cette tendance de «dédramatisation» ou de «diminution du 
caractère émotif " de la conscience environnementale ${ }^{44}$. Particulièrement forte entre 2000 et 2002, elle perd néanmoins de sa force par la suite, comme l'indiquent les résultats de l'enquête menée en $2004^{45}$.

Après avoir passé en revue l'état de la conscience environnementale en Allemagne depuis les années 1990 et jusqu'au milieu des années 2000, revenons à l'hypothèse de départ de cet article portant sur les fondements d'un modèle allemand qui aurait servis de modèle à la France. Selon une idée préconçue, les causes de cet intérêt seraient historiques et directement liées à une attitude de protection de la nature née à l'époque romantique. Pour Céline Caro qui analyse le développement de la conscience environnementale en Allemagne et en France dans une perspective historique, ce cliché se vérifie pour une période spécifique (les années 1980) mais ne permet cependant pas de conclure que les conditions historiques en Allemagne expliquent une plus grande sensibilité à ces questions ${ }^{46}$. L'auteure avance l'hypothèse, initialement formulée par Klaus Eder, que ces présupposées "racines historiques", considérées comme les fondements de cette conscience environnementale allemande, correspondraient à une construction, à un argument avancé uniquement pour justifier cette posture ${ }^{47}$.

\section{Discours écologiste, discours sur la protection de la nature : des orientations différentes}

19 L'une des pistes pour la déconstruction de cet argument identifiée par Céline Caro peut être pensée à travers l'étude séparée de l'émergence du discours écologiste portant explicitement sur "die Umwelt » (l'environnement) et de celui centré sur "Naturschutz » (la protection de la nature). Contrairement à l'idée selon laquelle les Allemands seraient précurseurs en matière d'écologie, le parti écologiste «Die Grünen » et le Ministère de l'environnement, de la protection de la nature et de la sécurité des réacteurs nucléaires ( Bundesministerium für Umwelt, Naturschutz und Reaktorsicherheit), respectivement créés en 1980 et 1986, apparaissent relativement tard sur la scène politique allemande ${ }^{48}$. En revanche, Tobias Chilla souligne que le discours écologique s'inscrit dès le début en cohérence avec les luttes sociales émergeant en Allemagne de l'ouest suite au mouvement contre-culturel :

«Avec un certain retard sur les débats de 1968 concernant les réformes sociales, le discours écologique a de plus en plus d'audience au cours des années 1970. Le ferment de ce nouveau mouvement environnemental, ce sont souvent les protestations contre les centrales nucléaires, mais la palette des thèmes est rapidement élargie. En Allemagne (occidentale) le discours de l'époque est marqué par les mots d'ordre "mettons fin à..., arrêtons le..., sauvons le..." [...]. Ces revendications sont en étroite relation avec les débats sur les limites de la croissance qui sont au même moment illustrées par les crises pétrolières et qui vont de pair avec des changements multiples de l'esprit du temps et du mode de vie. [...] Comme dans d'autres domaines politiques, des mouvements citoyens divers émergent dans la protection de l'environnement et adoptent une attitude critique par rapport à l'action de l'État (cela apparaît d'autant plus comme une nouveauté, qu'auparavant, la protection de l'environnement institutionnelle était largement en harmonie avec le secteur associatif) [...] $»^{49}$.

L'émergence de ce discours politique écologiste marque un tournant par rapport à un discours plus ancien portant plus explicitement sur la protection de la nature. Cette pensée se définit comme une réaction contre le tourisme de la Belle Époque ${ }^{50}$. En 1935, la 
loi sur la protection de la nature («Reichnaturgesetz ») institutionnalise ce mouvement visant à limiter « l'exploitation sans retenue des ressources».

21 Ce discours se maintient au-delà de l'émergence de groupes écologistes et trouve une continuité à travers le «cri d'alarme " jeté par le journal Der Spiegel en 1981dans un numéro dont la couverture attire l'attention sur les forêts menacées ("Waldsterben»). Comme dans d'autres pays touchés par l'étalement urbain, «le débat sur le dépérissement des forêts devient la métaphore d'une catastrophe mondiale de l'environnement [...] et influence surtout les domaines politiques de la protection de l'air et de la nature $»^{51}$. Dans ce contexte, on peut considérer que le discours alarmiste sur la disparition de la forêt allemande alimente le courant écologiste porteur des luttes sociales qui a émergé dans les années 1970.

Cet appel à réagir face à des problèmes relevant d'une catégorie désormais identifiée comme un «enjeu environnemental» est précisément ce qui fait écho chez les voisins européens : «Le fait que les journalistes en France ou en Angleterre aient repris à leur compte "le waldsterben" ou "the waldsterben" n'a pas contribué à modérer l'argumentation $»^{52}$. La sensibilisation contre la disparition des forêts symbolise désormais l'engagement écologiste d'une nation qui, vue de l'étranger, en devient le leader.

Pourtant, à la même époque, d'autres sujets environnementaux sont débattus dans d'autres pays, à l'instar du recul de la biodiversité, sans susciter pour autant une telle réappropriation en Allemagne et former les bases d'un modèle allemand en circulation dans les espaces publics des pays européens. Dans la République fédérale ${ }^{53}$ des groupes émergent revendiquant la « protection de la nature » dès le milieu des années 1950 :

« La pollution commence aussi à être du ressort des protecteurs de la nature. Leurs mouvements ont été regroupés en 1954 au sein du Kulturbund, qui rassemble des associations couvrant différents domaines: l'histoire, la philatélie, etc. Les naturalistes légitiment leur engagement dans la société socialiste en s'appuyant sur le concept de Landeskultur qui signifie, outre le respect des forces naturelles, la satisfaction des besoins d'une société humaine dans l'aménagement planifié d'une région $»^{54}$

Par ailleurs, on observe que ces engagements sont menés dans le cadre de mouvements sociaux au sein de l'Église. Ces mouvements plus ou moins ouvertement dissidents, ralliés à la cause de l'Eglise réformée au début des années 1980, sont directement inscrits dans une lutte qui dépasse les simples intérêts environnementalistes. Ces mobilisations acquièrent une dimension politique et sociale :

«Leur but est, à la fois, de combattre pour un meilleur environnement et pour la paix. À ce titre, ils sont utiles à la RDA qui se veut un État pacifiste face à l'Amérique de Reagan. Leur action ne vise pas à renverser le régime et reste purement locale. Cela ne les dispense pas d'être l'objet d'une surveillance renforcée de la part de la Stasi. Ces groupes cherchent d'abord à informer la population des risques inhérents à la pollution en tenant des réunions le dimanche et en éditant des journaux dans le cadre de l'Église. Ils tirent leurs informations de sources diverses: des médias de l'Ouest, dont les revues transitent clandestinement par la Hongrie, de quelques rares scientifiques $»^{55}$. 


\title{
3. Partis, syndicats, associations : les porteurs historiques du modèle allemand
}

\subsection{La dispersion des luttes sociales}

À travers la lecture que les auteurs français font du modèle allemand, on constate qu'ils se focalisent sur les institutions politiques, syndicales ou associatives qui se soucient d'une société «plus écologique ». Ce portage se manifesterait par une plus grande puissance d'action des associations précisément fondées sur la valorisation de la « conscience environnementale » et du parti écologiste «Die Grünen ». À cela s'ajoute la variété des acteurs et des instances de médiation s'appropriant les thèmes de l'environnement et de l'écologie en Allemagne: «acteurs non gouvernementaux et intervenants ", " groupes d'intérêt qui représentent des parts importantes de la société ", «think tanks qui se retrouvent près de la sphère politique tout en gardant leur indépendance $\aleph^{56}$. Dans la citation suivante Hartmut Marhold souligne d'une part la multiplicité des acteurs participant aux décisions gouvernementales en matière d'environnement et d'autre part le fait que ces questions font partie intégrante du champ du social :

\begin{abstract}
«En Allemagne, les gouvernements n'aiment pas prendre de décision sans avoir consulté tous ceux qui représentent les différents intérêts sociaux sans avoir fait des tentatives concrètes pour trouver des solutions acceptables pour tous les intervenants, sans chercher de plus larges majorités que celles issues des élections générales, sans avoir consulté des think tanks indépendants (souvent financés par le gouvernement lui-même) $\aleph^{57}$.
\end{abstract}

Pour Dany Trom, Loïc Blondiaux et Guillaume Coutry, la puissance des forces écologistes allemandes s'explique avant tout par la concentration de thématiques sociales dans le discours et les champs d'action de ces institutions, là où en France le développement d'une constellation des partis de gauche dans laquelle le socialisme aurait joué un rôle non négligeable aurait eu pour conséquence de disperser les luttes sociales. Si, dans l'espace public français, les questions liées à l'abolition de la peine de mort, aux droits des homosexuels et à la dépénalisation de l'avortement sont défendues par les différents partis de gauche, en Allemagne ces questions sont essentiellement intégrées aux luttes du mouvement écologiste puis du parti vert. Dans cette perspective, la capacité de l'écologie allemande à mobiliser une partie de la population autour des luttes sociales serait davantage due à l'histoire de la répartition des forces politiques dans ce pays qu'à l'existence d'un «modèle allemand ", culturellement et politiquement plus favorable aux questions écologiques émergentes.

\subsection{Une résistance française à l'écologie?}

Les trois auteurs précités décèlent en France une certaine résistance politique à l'écologie. Si le marxisme domine en Allemagne au début du siècle, cette posture s'inverse après la création de la RFA. En France, en revanche, «le PC est puissant, canalise les velléités révolutionnaires, se trouve renforcé par l'adhésion massive des intellectuels au marxisme (en particulier dans sa version althussérienne) $\aleph^{58}$. La gauche française devra attendre les années 1960 et l'émergence d'une « deuxième gauche ", plus sceptique vis-àvis du rôle de l'État, anti-productiviste, prenant le chemin qu'avait emprunté quarante 
ans plus tôt l'intelligentsia de gauche sous la République de Weimar. Cette nouvelle gauche se nourrit d'une critique éthique et moralisante du capitalisme, imprégnée des apports du tiers-mondisme et constitue, pour ces auteurs, le terreau fertile de l'écologie politique, dont les formes néo-rurales et les combats anti-nucléaires restent néanmoins secondaires dans le champ des luttes sociales françaises.

De la même façon, une résistance politique à l'écologie en France s'expliquerait aussi par des divisions au sein même des écologistes. Une hétérogénéité idéologique des différents courants repousserait, selon Céline Caro, « la création d'une formation politique unique " 59. En raison de « l'espoir d'une alternance politique en faveur de la gauche en 1981 et les appels du Parti socialiste à partir de 1977 », ces courants auraient hésité avant de créer leur propre parti. ${ }^{60}$.

\subsection{L'écologie politique allemande : faire la société plutôt que s'opposer}

29 Si les auteurs français se tournent vers l'écologie politique allemande pour nourrir la réflexion sur les « lacunes » apparentes du cas français, il n'en demeure pas moins que les textes témoignent d'un regard critique porté sur ce modèle. En 2007, dans son article portant sur l'histoire comparée des partis verts allemand et français, Jean-Paul Gaudillière relève la capacité des «Grünen» à porter les luttes sociales et environnementales en faisant consensus, sans parvenir à constituer une force d'opposition :

«L'écologie politique allemande partage nombre de traits avec son homologue française. Pourtant, même si les Grünen, depuis leur passage au gouvernement et l'alliance avec la social-démocratie, connaissent aussi des difficultés à définir une stratégie, ils restent le premier parti écologique européen [...]. Pour nombre de ses cadres, la condition de cette réussite a été l'effacement de l'identité " gauchiste ". Au milieu des années 1990, Joschka Fischer imaginait sans états d'âme faire des Grünen un " FDP écologique " ${ }^{61}$.

À l'inverse, Pierre Jacquiot estime que la capacité de consensus des "Grünen ", notamment par rapport aux questions économiques, explique en partie leur succès auprès des militants. Selon lui, « les Allemands militent pour un environnementalisme économiquement viable ", ce qui expliquerait que l'écologisme se soit davantage répandu dans la société allemande ${ }^{62}$. De même, du côté des associations, on constate une propension au consensus. Par exemple, Greenpeace Deutschland se montre prêt à coopérer avec les industriels en participant à des expertises en qualité d'observateur afin de développer des produits et des stratégies plus respectueux de l'environnement. Ils abandonnent ainsi les attitudes de « confrontation intransigeante ${ }^{63}$.

31 À travers ces analyses des formes institutionnelles du politique en Allemagne, les auteurs précités soulignent l'existence d'une « conscience environnementale » ainsi que d'actions pouvant être qualifiées de " comportement environnementaliste ». Ces phénomènes ne s'expliquent pas par une prétendue culture romantique soucieuse de la préservation de la nature allemande, mais par une configuration politique dans laquelle la multiplicité des forces politiques et la formulation convergente des catégories sociales et environnementales donne davantage de poids aux problèmes écologiques. Outre cette configuration politique, un "développement de l'écologie au sein de l'espace public, soutenu par une médiatisation et une communication spécifique" constitue une 
explication de cet engagement tel qu'il est observé en Allemagne ${ }^{64}$. Cette explication serait plus plausible que les supposées racines historiques du développement d'une conscience environnementale allemande.

\section{Conclusion}

32 À la fin de son article portant sur les idées reçues relatives à l'imaginaire de la nature en Allemagne, Tobias Chilla analyse l'émergence du réseau européen de protection de la faune Natura 2000 (FFH) :

« Du point de vue qualitatif le processus FFH représente aussi une césure, celui du changement d'échelle pour aller d'une perspective régionale et nationale à la réflexion à l'échelle européenne. Cela a pour conséquence de le placer au centre des préoccupations écosystémiques des parties de nature qui auparavant, n'étaient pas considérées comme devant être particulièrement protégées (parce qu'elles n'étaient ni rares, ni spectaculaires). [...] Le processus FFH se caractérise surtout par son insertion dans la nouveauté à partir des différentes voies suivies pour la mise en place des directives. $" 1$.

Ainsi, l'auteur montre que l'Europe et non le « modèle allemand » a introduit de nouvelles logiques en matière de "protection de la nature ", dans sa dimension territorialisée. L'élargissement des frontières et des domaines de compétence de l'UE, mais aussi la circulation des pratiques et des représentations permettent le transfert des modèles et la perméabilité des manières de faire et de voir ainsi que des actions et des territoires.

De la même manière, Céline Caro suggère que l'échelle européenne exerce une influence importante sur les perceptions des enjeux environnementaux en France et en Allemagne. L'écart entre les deux pays en matière de préoccupations environnementales, très important dans les années 1980 et supposé trouver son origine dans le «modèle allemand ", diminue en raison des politiques environnementales européennes ${ }^{66}$. Sans constituer un facteur explicatif unique, cette piste de l'européanisation des pratiques et des représentations ayant trait à l'engagement politique en faveur de l'environnement permet de s'éloigner d'une analyse culturaliste. L'Allemagne se démarque en termes de " conscience environnementale ", du moins dans la sphère des institutions associatives et politiques pour deux raisons principales. D'une part, les luttes sociales croisent les thématiques environnementales; d'autre part ces collectifs, à la fois plus nombreux et plus diffusés dans toutes les strates de la société, participent davantage à la prise de décision politique. Dans ce domaine, les revendications de type écologiste doivent donc être analysées en parallèle et dans la complexité de leurs relations avec d'autres sujets de mobilisation caractéristiques de la seconde moitié du XXe siècle. Le "modèle allemand ", porteur d'une histoire en termes de "protection de la nature", s'est ancré dans l'organisation de la société et dans les luttes qui la construisent pour acquérir une légitimité qui dépasse les frontières. 


\section{NOTES}

1. Les références bibliographiques ont été choisies en fonction de leur degré de pertinence dans les bases de données francophones Cairn et Persée, à partir de recherches comportant les motsclés suivants : France, Allemagne, écologie, environnement, développement durable.

2. Danny Trom, Loïc Blondiaux, Guillaume Courty, « Le Parler Vert : réflexion sur les structures discursives de l'idéologie écologiste ", Politix, n 9, vol. 3, 1990.

3. Comme la partie 1.1 de cet article le montrera, Jean-Paul Bozonnet distingue environnementalisme et écologisme par rapport au lien avec l'action et l'engagement politique : alors que l'écologisme a pour trait essentiel l'engagement politique actif face aux enjeux environnementaux (prenant ainsi la forme d'une idéologie au même titre que les autres idéologies politiques (libéralisme, communisme, etc.) ou religieuses), cette dimension est absente de la notion d'environnementalisme. Jean-Paul Bozonnet, «Les convictions écologistes en Europe: les acteurs et leur vision de monde» in: Jean-Paul Bozonnet, Joel Jakubec (dir.), L'écologisme à l'aube du XXI ${ }^{e}$ siècle, Genève : Georg Editeur, 2000, p. 185-213.

4. Hélène Blanc, Michel Bodet, «La décroissance : traduction et représentation en Allemagne ", 2012 (article en ligne : http://www.unicaen.fr/colloques/cnriut2011/papers/160.pdf), p. 3 : « En Allemagne, aucun terme ne s'est jusqu'à présent imposé. En l'absence de mot accepté par tous, certains mouvements militants, comme ATTAC Deutschland, utilisent parfois dans leurs textes le mot français "décroissance". Ils ont pourtant proposé quelques mots allemands pour traduire cette idée :

- "das Entwachstum" est le mot le plus riche sémantiquement. "Wachstum" signifie "croissance". Le préfixe "ent" a deux sens différents : il marque soit l'opposition, soit la séparation ("entwachsen" prend alors le sens de "s'échapper du domaine de la croissance", "abandonner l'idée de croissance"). Dans d'autres contextes, "entwachsen" peut signifier "dépasser quelque chose, sortir d'une phase", ici "dépasser le stade de la croissance". "Ent" correspond dans certains verbes au préfixe français "dé" (falten : plier, entfalten : déplier ;entkoloniarisieren : décoloniser).

- "die Wachstumsverweigerung": le refus de la croissance, l'objection de croissance. Ce terme évoque pour les Allemands le mot "Kriegsdienstverweigerung", objection de conscience. Le combat contre le "dogme" de la croissance est ainsi assimilé à une révolte morale contre un dogme qui mine les fondements éthiques de la société.

- "die Postwachstumsökonomie" :l'économie d'"après la croissance", comme si la croissance avait été une phase (peut-être nécessaire, pour certains) dans l'évolution de l'humanité, mais qu'il convient aujourd'hui de dépasser et de remplacer.

- "die Wachstumsrücknahme" : attitude volontaire d'inflexion du processus de croissance. Il s'agit ici de ralentir la croissance, sans néanmoins rompre radicalement avec l'idée.

- On trouve aussi en allemand l'expression "jenseits des Wachstums", au-delà de la croissance. Elle semble traduire la volonté de penser l'économie et la société sans prendre la notion de croissance comme référence absolue, comme condition préalable à tout progrès et à toute évolution positive.

Dans tous les termes allemands que nous avons recensés, le mot Wachstum est associé à un terme négatif ou restrictif. Pour que le mot composé ait un sens positif, il est nécessaire que Wachstum soit connoté négativement ».

5. À propos de la «construction sociale de la nature » dans le contexte allemand, Tobias Chilla précise: "Suivant cette perspective, la "nature" ne peut pas être considérée de manière objective, quasiment pour "elle-même". Elle ne peut être regardée qu'en rapport avec le contexte 
social [...]. La nature est ainsi le résultat d'interprétations sociales et de jugements et, en ce sens, elle est "construite". Cela vaut aussi pour la protection de la nature : bien que celle-ci soit depuis quelque temps considérée comme une affaire de science naturelle, la décision de ce qu'il est valable de protéger est toujours étroitement reliée avec les interprétations sociales [...]. Dans ce sens, la "construction" n'est pas à comprendre comme une préférence, mais plutôt comme une tentative d'intégrer les processus sociaux» (Tobias Chilla, "La protection de la nature en Allemagne - une nature allemande?", Revue Géographique de l'Est, n 47, 2007, p. 2-9 ; ici p. 2. Voir aussi : Florence Rudolf, L'environnement une construction sociale, pratiques et discours sur l'environnement en Allemagne et en France, Strasbourg : Presses Universitaires de Strasbourg, 1998).

6. Lionel Charles, Bernard Kalaora, «Sociologie et environnement en France. L'environnement introuvable?", Écologie \& Politique, $\mathrm{n}^{\circ}$ 27, 2003, p. 31-57.

7. Tobias Chilla, art. cit. Une grande exposition sur l'art en Allemagne de 1800 à 1930, «De l'Allemagne ", proposée au Louvre du 28 mars au 14 juin 2013, a suscité une polémique dans les deux pays, en référence au lien établi entre cette "idéalisation de la nature romantique » et la montée du national-socialisme.

8. Ibid., p. 2

9. Cette valorisation est décrite de manière différente par plusieurs auteurs : Pierre Jacquiot la présente comme une "différence de force» des associations écologistes en France et en Allemagne, mesurée par «le nombre d'adhérents, de militants, de salariés » et les ressources financières mobilisées dans la "protection de l'environnement", au sein d'associations écologistes (Pierre Jacquiot, «La normalisation de l'écologisme : comparaison des associations vertes en France et en Allemagne » in : Jean-Paul Bozonnet, Joel Jakubec (dir.), L'écologisme à l'aube du XXI siècle, Genève : Georg Editeur, 2000, p. 149-183). Pour Céline Caro, il s'agit d'une différence de "degré » du développement de l'écologie comme pensée, plus aboutie en Allemagne qu'en France en raison du fort engouement qu'elle suscite à partir de la seconde moitié du XXe siècle (Céline Caro, Le développement de la conscience environnementale et l'émergence de l'écologie politique dans l'espace public en France et en Allemagne, 1960-1990, Paris/Dresden: Université Sorbonne Nouvelle - Paris III, Technische Universität Dresden, 2009, p. 454). Cette valeur s'estimerait, pour l'auteur, à travers la «montée des préoccupations environnementales» et par le "développement de discours d'écologie politique» (ibid.). Enfin, un troisième mode d'interprétation est formulé par Annette Lang et porte sur l'importance de «l'influence politique » des associations écologistes en Allemagne, lesquelles ont joué un rôle important dans la formation de l'opinion publique en matière d'écologie et d'environnement. Outre ces trois différenciations d'ordre quantitatif, il en existe d'autres d'ordre qualitatif: la protection de l'environnement tout comme le thème de la nature « sauvage » sont au centre des discussions en Allemagne, alors qu'elles restent marginales en France (Annette Lang, Ist Nachhaltigkeitmessbar?, Stuttgart: ibidem-Verlag, 2003, p. 49-50). De la même façon, les Agendas 21 locaux sont plus diffusés du côté allemand (Hans Günther Barth, Annette Lang, Nachhaltige Stadtentwicklung in Deutschland und Frankreich, Frankfurt am Main/Berlin/Bern/Bruxelles/New York/Oxford/Wien : Peter Lang, 2003, p. 19).

10. Cette contribution s'inscrit dans une réflexion commune, menée dans le cadre du réseau de recherche « Saisir l'Europe - Europa als Herausforderung ». Le volet que nous abordons concerne la crise écologique et le développement durable, analysés à partir d'une approche critique.

11. La sélection des articles s'est effectuée selon une recherche par mots clés sur les moteurs de recherche francophones, à partir des termes «environnement - France - Allemagne» et "écologie - France - Allemagne ». Les thèmes du développement durable et de la durabilité, pourtant évoqués dans l'article, seront traités de manière secondaire.

12. Les chercheurs français ont souvent exclu l'environnement du champ des luttes sociales, ce qui explique en partie le manque d'intérêt pour les travaux portant spécifiquement sur l'environnement en sociologie. 
13. Jean-Paul Bozonnet, «Les convictions écologistes en Europe : les acteurs et leur vision de monde », art. cit., p. 186. Jean-Paul Bozonnet parle de « convictions écologistes ». Il accepte aussi la différence entre «écologisme » et "environnementalisme » et se concentre sur le premier terme.

14. Pierre Jacquiot, art. cit., p. 149.

15. Céline Caro, op. cit.

16. Peter Preisendörfer, Umwelteinstellungen und Umweltverhalten in Deutschland, Opladen : Leske et Budrich,1999, p. 17.

17. Ibid., p. 19.

18. Ibid., p. 19.

19. Jean-Paul Bozonnet, «De la conscience écologique aux pratiques. Comment expliquer le hiatus entre attitudes environnementalistes et les comportements ", in : HAL-SHS,

http://halshs.archives-ouvertes.fr/view_by_stamp.php?

\&halsid=a5l0157ben5f0s5mm5ppjj3ir6\&label=SHS\&langue=fr\&action_todo=view\&id=halshs-00160092\&version=1 (2007; consulté le 15.06.2013), p. 1.

20. Peter Preisendörfer, Umwelteinstellungen und Umweltverhalten in Deutschland, op. cit.,p. 19.

21. Ibid., p. 20.

22. Jean-Paul Bozonnet, «Les convictions écologistes en Europe : les acteurs et leur vision de monde », art. cit., p. 189, 194 et 197.

23. Ibid., p. 187.

24. Ibid., p. 194.

25. Ibid., p. 194.

26. Ibid., p. 197.

27. Mené sur la base d'entretiens semi-directifs et téléphoniques, le protocole d'enquête avait intégré les différences entre les anciennes Allemagne de l'est et l'ouest et comportait les thèmes suivants : les perceptions et connaissances de la population autour des enjeux environnementaux (a), leurs attitudes et comportements de manière générale (b) et plus particulièrement par rapport aux pratiques d'achat et de consommation (c), aux déchets et au recyclage (d), aux économies d'énergie et à la consommation d'eau (e), au transport et à l'automobile (f), à l'engagement public pour la protection de la nature et l'environnement $(\mathrm{g})$, et enfin leur perception des politiques institutionnelles et de leurs acteurs (h) (Peter Preisendörfer, op. cit., p. 21-22).

28. Ibid., p. 27-29.

29. Ibid., p. 31.

30. Ibid. p. 34-35.La dernière observation concernant l'amélioration de la perception des lois environnementales par la population est relative : la perception est en 1998 plus positive qu'en 1991. Cependant, une grande partie de la population considère qu'il n'y a pas assez de lois de protection de la nature ou de réglementation face aux enjeux environnementaux urgents et que les lois existantes ne sont pas assez respectées.

31. Par ailleurs, cette tendance positive n'est pas générale : nombreux sont ceux qui se montrent réticents à utiliser les transports en commun, à payer davantage d'impôts ou à respecter des restrictions dans la circulation des voitures. (Ibid., p. 37).

32. Ibid., p. 39-40.

33. Ibid., p. 55.

34. Ibid., p. 44.

35. Ibid., p. 55.

36. Ibid., p. 71.

37. Heiko Grunenberg, Udo Kuckartz, Umweltbewusstsein im Wandel,Opladen: Leske, Budrich, 2003 ; Udo Kuckartz, Anke Rheingans-Heintze, Trends im Umweltbewusstsein, Wiesbaden: VS Verlag, 2006. 
38. Heiko Grunenberg, Udo Kuckartz, Umweltbewusstsein im Wandel, op.cit., p. 16.

39. Les premières études de l'UBA datent des années 1990.

40. Ibid., p. 13 ; p. 31-37.

41. Ibid., p. 13.

42. Udo Kuckartz, Anke Rheingans-Heintze, Trends im Umweltbewusstsein, op. cit., p. 11-12, 18.

43. Ibid., p. 18.

44. Heiko Grunenberg, Udo Kuckartz, Umweltbewusstsein im Wandel, op.cit., p. 16.

45. Udo Kuckartz, Anke Rheingans-Heintze, Trends im Umweltbewusstsein, op. cit., p 27.

46. Céline Caro, op. cit., p. 467.

47. Ibid., p. 463-464.

48. Du moins par rapport à la France. Le Mouvement écologiste, devenu par la suite le parti des Verts puis Europe Ecologie - Les Verts, a été fondé en 1974 et marqué par la candidature de René Dumont à la présidentielle. Le premier Ministère de l'environnement, aujourd'hui renommé Ministère de l'écologie, du développement durable et de l'énergie, a été créé en 1971.

49. Tobias Chilla, art.cit., p. 4.

50. Ibid.

51. Ibid., p. 6.

52. Ibid., p. 5

53. Cet article n'a pas pour objectif d'entrer dans les détails des trajectoires des notions liées à l'écologie et à l'environnement en ex-RDA. On trouvera des références historiques précises de ces évolutions dans l'article de Michel Dupuy, «Les scientifiques et le politique : l'exemple de la pollution atmosphérique en ex-RDA (1949-1989) », Nature, sciences, société, n 12, 2004.

54. Ibid., p. 329.

55. Ibid., p. 334.

56. Hartmut Marhold, «Le développement durable - un principe directeur de la politique allemande? ", L'Europe en Formation, n³ 352, 2009/2, p.108

57. Idem.

58. Danny Trom, Loïc Blondiaux, Guillaume Courty, art.cit., p. 48.

59. Céline Caro, op. cit., p. 458-459.

60. Ibid., p. 459.

61. Jean-Paul Gaudillière, «L'écologie politique peut-elle être populaire ? Les Verts, les Grünen et la gauche?», Mouvements, $\mathrm{n}^{\circ} 50,2007$.

62. Pierre Jacquiot, art. cit., p. 176.

63. Ibid., p. 179.

64. Céline Caro, op. cit., p. 463.

65. Tobias Chilla, art. cit., p. 8.

66. Céline Caro, op. cit., p. 461.

\section{AUTEURS}

\section{ANAHITA GRISONI}

EHESS-CNRS 


\section{ROSA SIERRA}

Université Goethe de Francfort-sur-le-Main. Philosophe de formation, Rosa Sierra s'intéresse à la notion de durabilité, à son intégration dans les pratiques sociales et au rapport entre les questions de responsabilité et de justice telles qu'elles sont définies dans un contexte de crise écologique. Anahita Grisoni s'intéresse aux mouvements sociaux de résistance écologique nés dans le contexte de la construction de deux réseaux ferroviaires européens : la ligne à grande vitesse entre Lyon et Turin et la ligne Strasbourg-Kehl. Dans notre approche commune, nous abordons la crise écologique à la lumière d'une réflexion sur la crise en général, les croisements entre l'écologie et l'économie, tout comme entre l'écologie et la politique, et la manière dont les acteurs sociaux s'approprient ces thématiques. 